Trauma Berufskrankh 2014 - 16[Suppl 1]:13-15 DOI 10.1007/s10039-013-1974-1

Online publiziert: 17. August 2013

(c) Springer-Verlag Berlin Heidelberg 2013

M. Sczesny-Kaiser ${ }^{1} \cdot$ S. Lissek ${ }^{1}$ O. Höffken ${ }^{1} \cdot$ V. Nicolas ${ }^{2} \cdot$ R. Meindl ${ }^{3} \cdot$ M. Aach ${ }^{3}$

T.A. Schildhauer ${ }^{3} \cdot$ P. Schwenkreis ${ }^{1} \cdot$ M. Tegenthoff ${ }^{1}$

${ }^{1}$ Neurologische Klinik und Poliklinik, BG-Universitätsklinikum Bergmannsheil Bochum

${ }^{2}$ Institut für Radiologische Diagnostik, interventionelle Radiologie und Nuklearmedizin,

BG-Universitätsklinikum Bergmannsheil Bochum

${ }^{3}$ Chirurgische Klinik und Poliklinik, BG-Universitätsklinikum Bergmannsheil Bochum

\title{
Exoskelettales
}

\section{Rehabilitationstraining bei Querschnittgelähmten}

\author{
Fallserie zur neuronalen Plastizität
}

\section{Hintergrund und Fragestellung}

In den letzten 10 Jahren wurden motorbetriebene Gehorthesen entwickelt, um Patienten mit Paraplegie das Laufen zu ermöglichen. Eine besonders innovative Entwicklung sind Exoskelette, also von außen anliegende, motorbetriebene Stützorthesen. Beim HAL ${ }^{\circledR}$-Robot Suit (Cyberdyne, Japan) handelt es sich um ein solches Exoskelett, das noch vorhandene Willkürkontraktionen über Oberflächen-

\begin{tabular}{|l|l}
\hline \multicolumn{2}{|l}{ Abkürzungsverzeichnis } \\
\hline$A 1$ & $\begin{array}{l}\text { Amplitude des ersten Reizes des } \\
\text { Doppelreizes }\end{array}$ \\
\hline$A 2 s$ & $\begin{array}{l}\text { Amplitude des zweiten Peaks des } \\
\text { Doppelreizes nach Subtraktion }\end{array}$ \\
\hline$B A 1$ & Brodmann-Areal 1 \\
\hline$E E G$ & Elektroenzephalogramm \\
\hline$E M G$ & Elektromyographie \\
\hline$E N G$ & Elektroneurographie \\
\hline$f M R T$ & $\begin{array}{l}\text { Funktionelle Magnetresonanztomo- } \\
\text { graphie }\end{array}$ \\
\hline$H A L^{\bullet}$ & Hybrid Assistive Limb \\
\hline$k$ & Voxelschwellenwert \\
\hline$M E P$ & Motorisch evozierte Potenziale \\
\hline$M R T$ & Magnetresonanztomographie \\
\hline$S 1$ & Primär somatosensibler Kortex \\
\hline$S E P$ & Somatosensibel evozierte Potenziale \\
\hline
\end{tabular}

EMG-Elektroden registriert und diese Signale in Bewegungen umsetzt.

Aus fMRT-Untersuchungen ist bekannt, dass es nach Eintritt eines Querschnitttraumas zu drastischen Veränderungen der somatotopen Repräsentationen im S1 kommt. So verschiebt und vergrößert sich das Handrepräsentationsareal nach medial in Richtung der sozusagen verlorenen Fuß- und Beinrepräsentation („medial shift“; [2]). Diese Modifikationen sind von Änderungen der neuronalen Erregbarkeit begleitet $[1,4]$. Unklar ist bislang, welche zerebralen Reorganisationsphänomene durch ein exoskelettales Rehabilitationstraining induziert werden.

\section{Untersuchungsmethoden}

Wir untersuchten 7 Patienten mit chronischer inkompletter Paraplegie (6 Patienten mit Konus-Kauda-Läsion, 1 Patient mit thorakalem Querschnittsyndrom, Zeit seit dem Trauma $>12$ Monate), die an einem intensiven, physiotherapeutisch angeleiteten exoskelettalen Lokomotionstraining mit dem $\mathrm{HAL}^{\circledR}$-Robot Suit für 3 Monate im Rahmen eines individuellen Heilversuchs teilnahmen. Die dargestellten Daten stammen aus Beobachtungen einer Fallserie. Die durchgeführten neurologischen Untersuchungen dienten zur Überwachung der Therapie und deren Auswirkungen auf das periphere und zentrale Nervensystem und fanden vor und nach der Trainingsperiode statt.

Alle Patienten wurden elektrophysiologisch und magnetresonanztomographisch untersucht (ENG, Tibialis-SEP, MEP, Doppelpuls-SEP, fMRT). Wir nutzten eine spezielle EEG-Ableitung, das sog. Doppelpuls-Medianus-SEP, um die Erregbarkeit im S1 beidseits zu untersuchen [3]. Dabei wurden mit je 400 Reizen elektrische Einzel- und Doppelpulse über dem N. medianus am Handgelenk mit einer Frequenz von $3 \mathrm{~Hz}$ und mit der 2,5-fachen Intensität der sensiblen Wahrnehmungsschwelle appliziert. Die kortikalen EEG-Signale wurden über EEG-Oberflächenelektroden aufgezeichnet und offline ausgewertet. Als Messparameter diente die sog. Doppelpulssuppression (A2s/ A1-Amplituden-Verhältnis, - Abb. 1). Funktionelle MRT-Untersuchungen wurden durchgeführt, um kortikale Repräsentationsänderungen im Handareal des S1 festzustellen (3-T-MRT, Philips, Achieva X-Series). Während des Scans stimulierten wir taktil mittels eines AirpuffSystems simultan die 3 Phalangen des Zeigefingers an beiden Händen. Es wur- 


\section{Exoskelettale Neurorehabilitation}

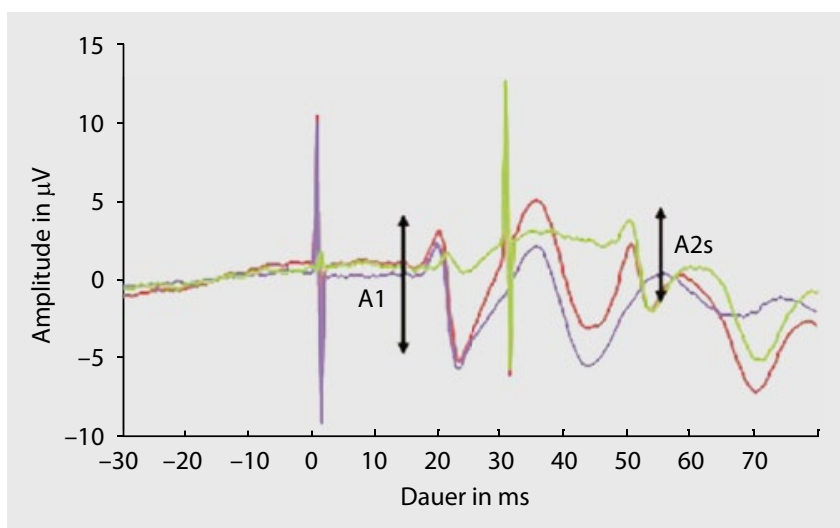

Abb. $1 \Delta$ Doppelpuls-Medianus-SEP mit Darstellung einer Einzelpulskurve (blau), einer Doppelpulskurve (rot) und der Subtraktion Doppelpuls-Einzelpuls (grün), Pfeile Amplituden A1 und A2s, Erläuterung der Abkürzungen s. Abkürzungsverzeichnis
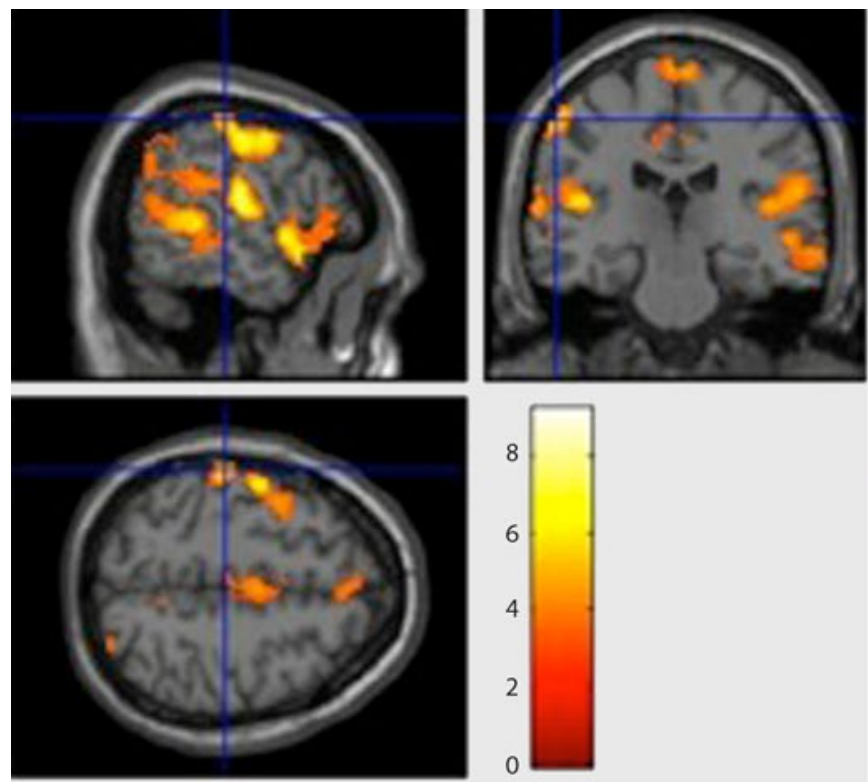

a Peak bei $-56-2452 \mathrm{BA} 1(\mathrm{p}<0,001 \mathrm{k}=10)$

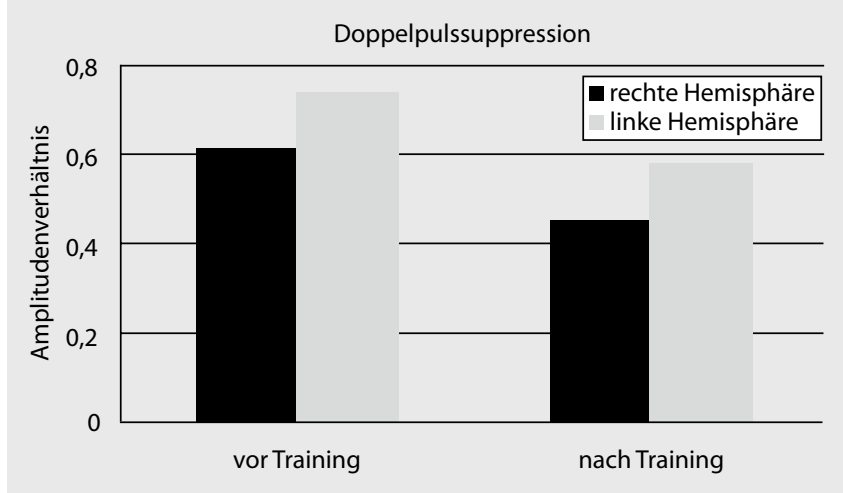

Abb. 2 \ Doppelpulssuppression: A2s/A1-Amplituden-Verhältnisse vor und nach dem Training jeweils für beide Hemisphären, Erläuterung der Abkürzungen s. Abkürzungsverzeichnis
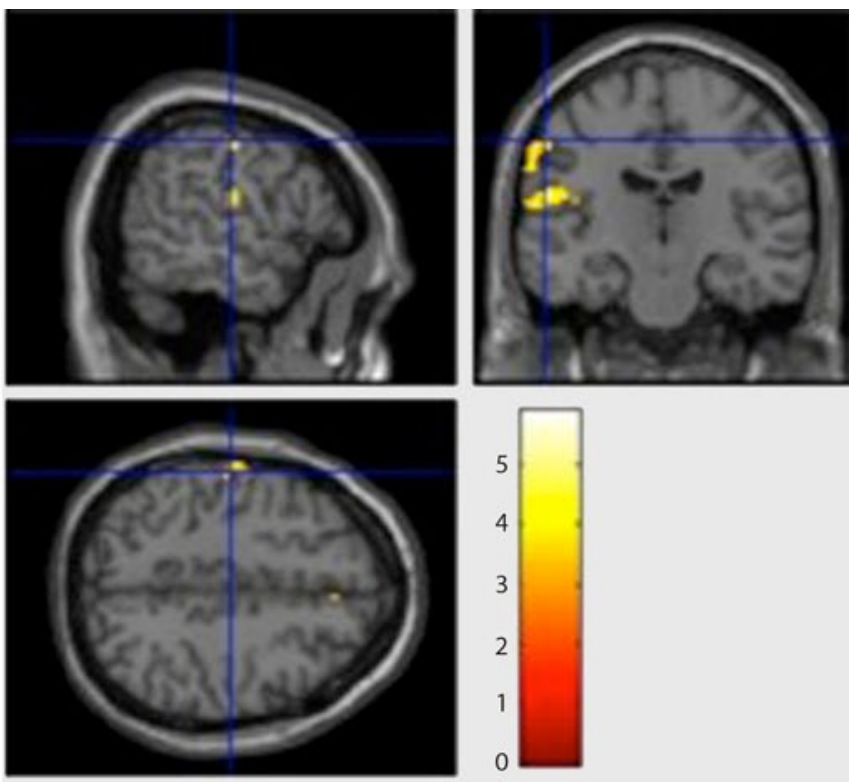

b Peak bei $-56-1846$ BA $1(p<0,001 k=10)$

Abb. 3 ム Funktionelle MRT, a vor, b nach Training, beispielhafte Daten eines Probanden („single-subject analysis“), Fadenkreuz im Aktivierungsmaximum des Handareals im S1 rechts (Stimulation linke Hand)

den 480 Stimuli mit einer Frequenz von 1,25 Hz appliziert.

\section{Ergebnisse}

Unsere Untersuchungen im Rahmen des individuellen Heilversuchs zeigen $3 \mathrm{Mo}$ nate nach dem Training mit dem $\mathrm{HAL}^{\oplus}$ Robot Suit eine erhöhte Doppelpulssuppression im S1, beidseits mittels Doppelpuls-SEP gemessen (• Abb. 2). Zusätzlich konnten wir eine reduzierte Aktivierung und eine laterale Verschiebung des Aktivierungsmaximums im S1 bei taktiler Stimulation des Zeigefingers feststel- len (• Abb. 3). Die übrigen elektrophysiologischen Untersuchungen blieben ohne signifikante Befundänderungen. Signifikante funktionelle Verbesserungen (Gehzeit, Gehstrecke, Gehgeschwindigkeit) wurden bei allen Patienten festgestellt (separate Untersuchungen).

\section{Diskussion}

Diese vorläufigen Ergebnisse aus individuellen Heilversuchen weisen auf plastische zerebrale Veränderungen hin. Die im Rahmen des Akutereignisses des Traumas stattgefundenen Reorganisations- phänomene mit „medial shift“, Vergrößerung der Repräsentation des Handareals [2] und Steigerung der Erregbarkeit [1] werden partiell aufgehoben. Diese Befunde weisen auf eine wieder zunehmende Repräsentation der unteren Extremitäten hin, die durch das Lokomotionstraining vermehrt benutzt werden und funktionelle Verbesserungen aufweisen. Die erneuten plastischen Veränderungen könnten somit als Abbild einer effektiveren Nutzung verbliebener intakter spinaler Neuronenverbindungen angesehen werden. Hierfür spricht, dass die standardelektrophysiologischen Messun- 
gen ohne Befundänderung blieben. Diese effektivere Nutzung ist wahrscheinlich für die funktionellen Fortschritte bei unseren 7 Patienten verantwortlich.

\section{Fazit für die Praxis}

- Exoskelettal unterstütztes Lokomotionstraining von Patienten mit chronischer Paraplegie stellt einen neuen innovativen Ansatz in der Neurorehabilitation dar.

- Es gibt Hinweise für zerebrale Reorganisationsphänomene, die zu funktionellen Verbesserungen führen oder Abbild derselben sind. Die rein deskriptiven Ergebnisse dieser Fallserie sollten in einer gesonderten Studie systematisch untersucht werden, um weitergehende Aussagen treffen zu können.

\section{Korrespondenzadresse}

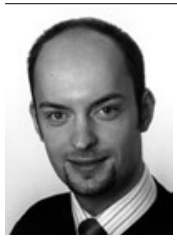

Dr. M. Sczesny-Kaiser

Neurologische Klinik und Poliklinik, BG-Universitätsklinikum Bergmannsheil Bochum, Bürkle-de-la-Camp-Platz 1, 44789 Bochum matthias.sczesny@ bergmannsheil.de

Interessenkonflikt. Der korrespondierende Autor gibt für sich und seine Koautoren an, dass kein Interessenkonflikt besteht.

The supplement containing this article is not sponsored by industry.

\section{Literatur}

1. Cohen LG, Roth BJ, Wassermann EM et al (1991) Magnetic stimulation of the human cerebral cortex, an indicator of reorganization in motor pathways in certain pathological conditions. J Clin Neurophysiol 8(1):56-65

2. Henderson LA, Gustin SM, Macey PM et al (2011) Functional reorganization of the brain in humans following spinal cord injury: evidence for underlying changes in cortical anatomy. J Neurosci 31(7):2630-2637

3. Höffken O, Tannwitz J, Lenz M et al (2013) Influence of parameter settings on paired-pulse-suppression in somatosensory evoked potentials: a systematic analysis. Clin Neurophysiol 124(3):574580

4. Lotze M, Schertel K, Birbaumer N, Kotchoubey B (2011) A long-term intensive behavioral treatment study in patients with persistent vegetative state or minimally conscious state. J Rehabil Med 43(3):230-236

Trauma Berufskrankh 2014 · 16[Suppl 1]:13-15 DOI 10.1007/s10039-013-1974-1

(c) Springer-Verlag Berlin Heidelberg 2013

M. Sczesny-Kaiser · S. Lissek · O. Höffken · V. Nicolas · R. Meindl · M. Aach · T.A. Schildhauer P. Schwenkreis · M. Tegenthoff

\section{Exoskelettales Rehabilitationstraining bei Querschnittgelähmten. Fallserie zur neuronalen Plastizität}

\section{Zusammenfassung}

Hintergrund. Es werden zunehmend mehr Neurorehabilitationsverfahren entwickelt. Exoskelettal gestütztes Lokomotionstraining wird z. B. bei Querschnittsyndromen eingesetzt. Im hierfür entwickelten $\mathrm{HAL}^{\circ}$-Robot Suit werden bioelektrische Signale zur Steuerung der Bewegungen verwendet.

Patienten und Untersuchungen. Trainingsinduzierte zerebrale und periphere Veränderungen im Rahmen eines individuellen Heilversuchs bei Patienten mit chronischer Paraplegie wurden untersucht. Hierzu trainierten 7 Patienten mit chronischer inkompletter Paraplegie täglich (5 Tage pro Woche) über 3 Monate mit dem HAL ${ }^{\circ}$-Robot Suit. Vor und nach dieser Trainingsperiode wurden elektrophysiologische Messungen und funktionelle Magnetresonanztomographieuntersuchungen (fMRT) durchgeführt, um zentrale und periphere Reorganisationsphänomene darzustellen.

Ergebnisse. Die Ergebnisse des DoppelpulsSEP (SEP: somatosensibel evoziertes Poten- zial) und der fMRT zeigten eine verminderte Erregbarkeit, eine verminderte Aktivierung, eine Verkleinerung und laterale Verschiebung der Handrepräsentation im primär somatosensiblen Kortex beidseits.

Schlussfolgerung. Die beschriebenen Ergebnisse weisen auf neuronale plastische Veränderungen im Gehirn hin, die zeitgleich zu den funktionellen Verbesserungen (Gehzeit, Gehstrecke, Gehgeschwindigkeit) der Patienten auftreten. Kortikale plastische Veränderungen führen scheinbar zu einer effektiveren Nutzung verbliebener intakter spinaler Neuronenverbindungen und somit zu den funktionellen Fortschritten. Diese Ergebnisse einer Fallserie sollten im Rahmen einer systematischen Studie überprüft werden.

\section{Schlüsselwörter}

Querschnittlähmung · Paraplegie ·

Somatosensorischer Kortex · Funktionelle Magnetresonanztomographie $\cdot$ Kortikale Plastizität

\section{Exoskeletal rehabilitation in chronic spinal cord injury. Case series for neuronal plasticity}

\section{Abstract}

Background. New tools for neurorehabilitation have been recently developed, including locomotor training with exoskeletons in spinal cord injury patients. The $\mathrm{HAL}^{\otimes}$ robot suit uses minimal bioelectrical signals from the patient to induce leg movement. Subjects and methods. In this case series, we looked for training-induced cerebral and peripheral reorganization phenomena in patients with chronic paraplegia. Over a 3-month period, 7 patients with chronic incomplete spinal cord injury participated daily (5 days/week) in $\mathrm{HAL}^{\bullet}$ exoskeleton-supported treadmill training. Prior to and after this training period, electrophysiological measurements and functional magnetic resonance imaging (fMRI) were performed to determine cortical and peripheral reorganization.

Results. We could show reduced pairedpulse inhibition, reduced activation, re- duced representation area, and a lateral shift of the activation maximum of the hand area in primary somatosensory cortex bilateral by means of paired-pulse SEP (somatosensory-evolked potential) of the median nerves and fMRI.

Conclusion. The results of our case series show plastic changes in the brain that accompany functional improvement. They sug gest that cortical plastic changes due to improved use of the remaining intact spinal connections, rather than regeneration of the lesioned spinal connections might be responsible for the functional improvement in our patients. The results of these 7 cases should be verified in a systematic clinical trial.

\section{Keywords}

Spinal cord injuries . Paraplegia .

Somatosensory cortex . Magnetic resonance imaging, functional $\cdot$ Cortical plasticity 